\title{
Research on Micro-Satellite Orbit Forecasting Technology Based on Artificial Intelligence
}

\author{
Haitao Yang ${ }^{1}$, Mingyong Jiang ${ }^{1}$, Yinan $\mathrm{Li}^{1}$, Hang Chen ${ }^{1, *}$ \\ 1 School of Space Information, Space Engineering University. \\ *corresponding author’s email: hitchenhang@foxmail.com
}

Keywords: Micro-Satellite, Orbit Forecasting, Artificial Intelligence

\begin{abstract}
Consider to the limitations of existing forecasting technologies and the characteristics of huge scale of micro-satellite network, we propose intelligent prediction technology of the orbit of MicroSatellite based on artificial intelligence in this paper. Some numerical simulations have been made to verify the proposed intelligent prediction technology. The experiments result shows that the mean square error of the result is lower than $2 \mathrm{~km}$.
\end{abstract}

\section{Introduction}

In order to study the promotion and application of low-orbit small satellite networking technology, countries have successively planned and carried out low-orbit small satellite networking projects to meet the real-time data transmission to the ground. In recent years, with the development of countries' plans, small satellites have developed explosively, and the number of orbits has increased year by year. Since 2013, the total number of small satellites in orbit has accounted for more than $60 \%$ of the total number of spacecraft in orbit during the same period.

At present, a large number of small satellites are operating in low-Earth orbit, which has aggravated the task of ground measurement and control and brought greater pressure to the management and control of microsatellites. Meanwhile, the space environment of low-Earth orbit is complex, and there will be great risks in the safe operation of small satellites in orbit. Therefore, exploring an intelligent satellite orbit management method is one of the important issues facing the aerospace field today [1-7].

This paper is organized as follows: Section 2 gives the principle of the proposed algorithm. Simulations are executed in section 3 and section 4 concludes this paper with a brief summary.

\section{Status and analysis}

\subsection{Micro-satellite Networking Status}

American Space X company "startlink" plans to launch 4,425 small satellites with an orbital height of 1110-1325KM, providing a wide range of broadband and communication services for users worldwide. The Boeing plans to launch 2956 small satellites with an orbital height of 1200KM and will use V-band to provide fixed satellite services. Samsung plans to launch 4,600 small satellites with an orbital height of 160-2000KM to provide data transmission services for users worldwide. Canadian Telesat plans to launch at least 117 small satellites and provide global network communications services to governments, military, enterprises and individuals through optical links between satellites. 


\subsection{Status of Small Satellite Orbit Forecast}

At present, the orbits of satellites are mainly forecasted by mathematical models combined with measurement and control data, which are roughly divided into three categories: numerical models, analytical models and semi-analytic models. North American Aerospace Defense (NORAD) provides two-line elements (TLE) combined with analytical models for orbit prediction is the mainstream method for satellite orbit prediction. This model includes a correlation model of the perceptual dynamics of lowEarth orbits, which has high prediction accuracy. NORAD's TLE needs to be calculated in the Simplified General Perturbation Version 4 (SGP4) analytical model. SGP4 is suitable for satellites with a near-Earth space target orbital period less than 225 minutes [1], and the prediction accuracy is generally in the tenkilometer level.

\subsection{Analysis}

The development of microsatellites is the basis for future low-orbit communication and global Internet construction. In order to ensure the safe operation of satellites of various systems in orbit, the demand for orbital forecasts will increase, and the accuracy requirements will increase. Existing forecasting models have complex calculation processes and need to wait for regularly updated TLE data to maintain forecasting accuracy. For the large number of tiny satellites, the predictions of traditional mathematical models are difficult to meet future developments in terms of accuracy and period.

\section{Requirements and measures}

\subsection{Requirements}

The complex environment of low-orbit space will cause the deviation of tiny satellite orbits and the risk of collision with other satellites. At the same time, a large amount of space debris in low-orbit space threatens the safe operation of this tiny satellite.

At present, a large number of tiny satellites are pouring into near-Earth space, causing a sudden increase in the workload of ground measurement and control stations and a sharp increase in the workload of orbital forecasting. Due to the large number, small size, and fast transit speed, the existing methods cannot track the orbit of microsatellites in an all-round way, which brings great challenges to the management and control of microsatellites.

In order to solve the practical dilemma and improve the management and control effect, it is necessary to implement intelligent management and control of small satellite operations, and to timely evaluate and warn the risks of satellites to ensure the safe operation of the small satellites after networking. The most important thing is to be able to accurately predict the movement position of each satellite.

\subsection{Measures}

Artificial intelligence is widely used in various fields, and related recurrent neural networks have strong nonlinear learning and processing capabilities. Through the study of historical data, you can grasp the change laws in the data and forecast the time series data. This provides a good idea for solving the precise prediction of low-orbit micro satellite orbits.

\section{$1:$ Tool}

Long Short-Term Memory Neural Network (LSTM) was proposed by Sepp Hochreiter and Jurgen Schmidhuber in 1997. It is a new type of deep learning neural network based on recurrent neural networks. The main purpose is to establish a long-term dependence model to determine the optimal time delay of time series data. A neural network model suitable for long-term sequence data training and 
prediction.

The LSTM neural network consists of an input layer, a hidden layer, and an output layer. It differs from recurrent neural networks in that the hidden layer contains a storage module that can store information. The storage unit in the storage module has the capability of self-connection, adaptive and logic gate control information flow. There are two logic gates at the input and output layers to control the activation and call of the memory module by the input and output layers, respectively. The core of the storage unit is a linearly connected linear unit called a self-connected memory cell (Constant Error Carousel, CEC). Due to the existence of self-connected memory units, logic gates can be opened and closed autonomously through learning, so the problem of gradient disappearance in RNN neural networks has been solved. The specific network structure is shown in the figure 1 .

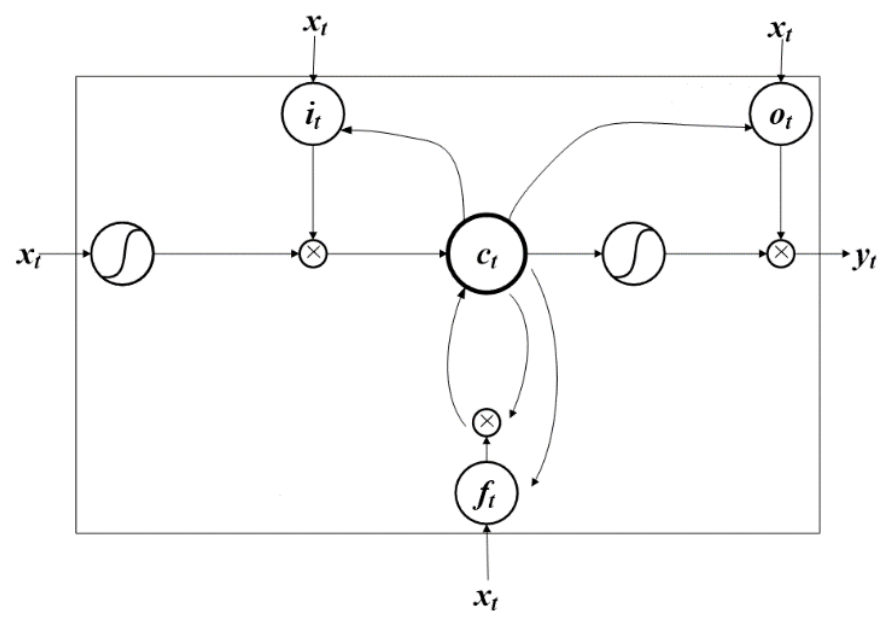

Fig 1 The specific network structure

In the figure, the input timing is set as $x=\left(x_{1}, x_{2}, \ldots x_{T}\right)$, and the output timing is set as $y=\left(y_{1}, y_{2}, \ldots y_{T}\right)$, where the forecast sequence is $T$. When predicting orbital data, the input is $\mathrm{X}, \mathrm{Y}$, and $\mathrm{Z}$ axis data in the geocentric inertial system. Output $\mathrm{X}, \mathrm{Y}, \mathrm{Z}$ data for subsequent time. The main purpose of the LSTM neural network is to predict the next time series data under the condition of a priori information, not to predict the data through how many steps. To achieve this, the input time series data will be iteratively calculated by the following formula [2]:

$$
\begin{aligned}
& i_{t}=\sigma\left(W_{i x} x_{t}+W_{i m} m_{t-1}+W_{i c} c_{t-1}+b_{i}\right) \\
& f_{t}=\sigma\left(W_{f x} x_{t}+W_{f m} m_{t-1}+W_{f c} c_{t-1}+b_{f}\right) \\
& f_{t}=\sigma\left(W_{f x} x_{t}+W_{f m} m_{t-1}+W_{f c} c_{t-1}+b_{f}\right) \\
& c_{t}=f_{t} \times c_{t-1}+i_{t} \times g\left(W_{c x} x_{t}+W_{c m} m_{t-1}+b_{c}\right) \\
& m_{t}=o_{t} \times h\left(c_{t}\right)
\end{aligned}
$$

In the LSTM neural network, three gates are first constructed to control the flow of information. ${ }^{{ }_{t}}$, $f_{t}, o_{t}$, Are input gate, forget gate, and output gate, respectively. Different gates have different effects on the control of information flow., The details are as follows [3]: 
Input gate ${ }^{i_{t}}$ : mainly controls the amount of information flowing into the memory cell;

Forget gate $f_{t}$ : It mainly controls the amount of memory cells accumulated at the previous moment to the memory cells of the current moment;

Output gate ${ }^{o_{t}}$ : It mainly controls the information flow in the memory cell at a certain time to flow into the current hidden state.

2: Experiment process

We mainly uses this LSTM neural network model for forecasting, The 100-day orbit data calculated by the HPOP model in the STK software is used as the sample data. The reference system uses the geocentric inertial Cartesian coordinate system (X, Y, Z) axis to express satellite orbit data. TacSat2 American scientific imaging satellite is selected as the research object.

In order to facilitate the mathematical description of the training phase and the forecast phase, first define an expression model, through which the state quantity of training samples:

$$
\vec{A}=(a: b, c)^{x x}
$$

Where $\vec{A}$ is the state quantity name, $X X$ is the model, $a$ is the sampling interval of the sample data, $b$ is the starting sampling time point of the sample data, and $c$ is the ending time point of the sample data sampling. The forecast model is shown in Table 1 and 2.

Table 1 Training phase

\begin{tabular}{cc}
\hline & Training phase \\
\hline Training samples & Implicit relationships in neural network training \\
$\left(T: T_{0}, T_{1}\right)^{H P O P}$ & $W_{1}=f_{1}()$ \\
$\left(T: T_{0}, T_{1}\right)^{H P O P}$ & $W_{2}=f_{2}()$ \\
$\left(T: T_{0}, T_{1}\right)^{H P O P}$ & $W_{2}=f_{3}()$ \\
\hline
\end{tabular}

Table 2 Forecast stage

\begin{tabular}{cccc}
\hline \multicolumn{4}{c}{ Forecast stage } \\
\hline Neural network input & Forecasting model & Forecast duration & Neural network output \\
(T: T0, T1)HPOP & W1 & T2-T1 & X(T: T1, T2)NN \\
$(\mathrm{T}:$ T0, T1)HPOP & W2 & T2-T1 & Y(T: T1, T2)NN \\
(T: T0, T1)HPOP & W3 & T2-T1 & Z(T: T1, T2)NN \\
Azimuth beamwidth $\left(^{\circ}\right)$ & 1.30 & Scene size $(\mathrm{m} \times \mathrm{m})$ & $200 \times 200$ \\
Angle 1 $\left(^{\circ}\right)$ & 21.00 & Angle 2 $\left(^{\circ}\right)$ & 66.00 \\
Angle 3 $\left(^{\circ}\right)$ & 111.00 & Angle 4 $\left(^{\circ}\right)$ & 156.00 \\
Angle 5 $\left(^{\circ}\right)$ & 201.00 & Angle $6\left(^{\circ}\right)$ & 246.00 \\
Angle 7 $\left(^{\circ}\right)$ & 291.00 & Angle $8\left(^{\circ}\right)$ & 336.00 \\
\hline
\end{tabular}

3: Comparative analysis

According to the LSTM neural network model, the data for 5 days, 10 days, and 20 days were forecasted for comparison. The $\mathrm{X}$-axis forecast data is mainly selected for detailed comparison with the sample data, as shown in the figure 2 : 

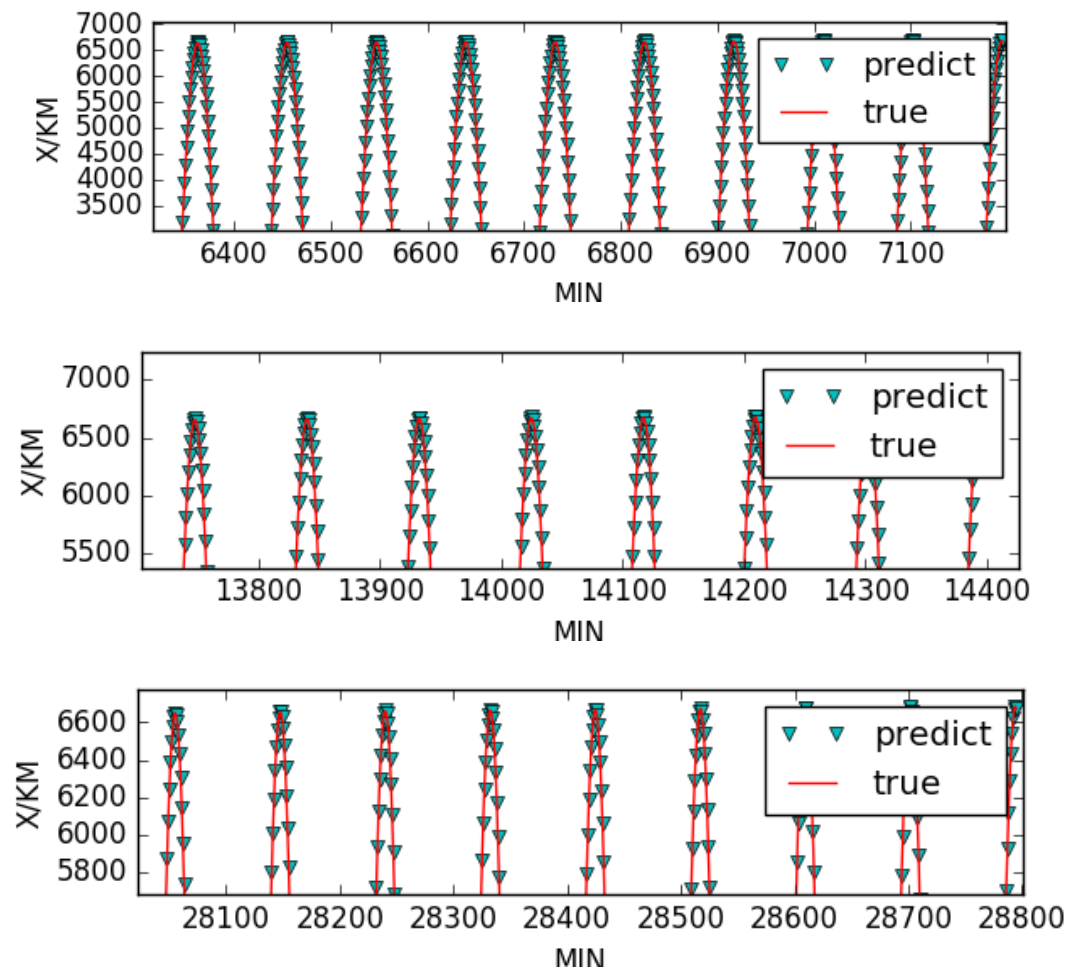

Fig 2 The $\mathrm{X}$-axis forecast data is mainly selected for detailed comparison with the sample data

In order to better show the variation of the forecast error range, the error of the forecast data and the sample data are compared in this paper. As shown in the formula:

$$
e=\bar{x}-x
$$

Where $\mathrm{e}$ is the error value, $\mathrm{x}^{\wedge}$ is the predicted value, and $\mathrm{x}$ is the sample value.

Calculate the error between the forecast data and the sample data for 5 days, 10 days, and 20 days, as shown in the figure 3 :
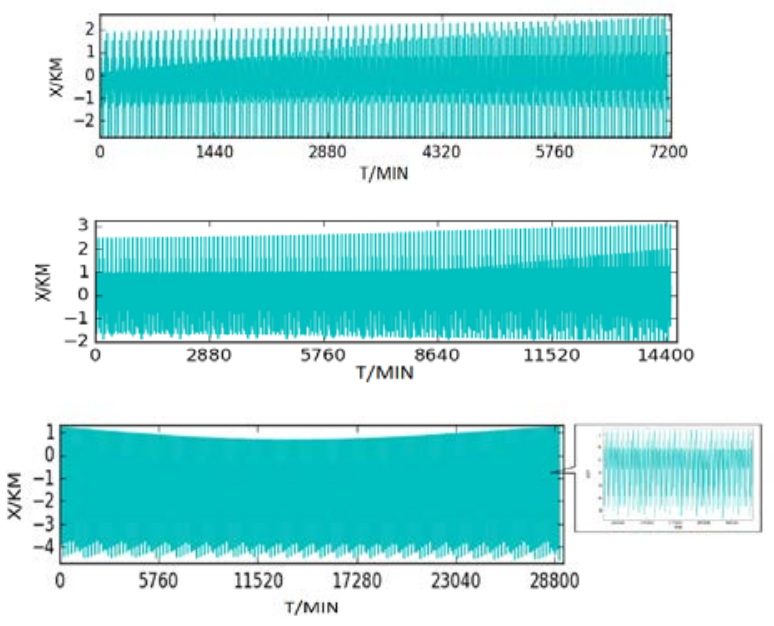

Fig 3 Calculate the error between the forecast data and the sample data for 5 days, 10 days, and 20 days

Since the mean square value is usually used to characterize the error distribution, it has a good 
convincing effect. Therefore, in the analysis of the prediction error of the micro satellite orbit, the mean square value of the error is used for analysis. The calculation formula is shown:

$$
\text { MSE }=\frac{1}{M} \sum_{i=1}^{M}(x-\bar{x})^{2}
$$

In the formula, $\mathrm{M}$ is the number of points participating in the calculation in a specific time period, $\mathrm{x}$ is the real value, and $\mathrm{x}^{\wedge}$ is the predicted value.

Calculate the mean square error of the $\mathrm{X}$-axis for 5 days, 10 days, and 20 days:

Table 3 Mean square error of the $\mathrm{X}$-axis for 5 days, 10 days, and 20 days

\begin{tabular}{cccc}
\hline \multicolumn{4}{c}{ Mean square error } \\
\hline Time/DAY & 5 & 10 & 20 \\
X-axis MSE/KM & 1.04 & 1.13 & 1.50 \\
\hline
\end{tabular}

After comparative calculations, the mean square error of the orbit error predicted by the neural network does not exceed 2KM. The short-term forecast is better than the long-term forecast, but the data error of the long-term forecast is still small, and the accuracy is greatly improved compared with the traditional mathematical model.

\section{Conclusion}

Focus on the problem of accurate prediction of micro satellite orbits, this paper uses artificial neural networks to forecast and analyze time series data. In the proposed method, the error value is significantly lower than the traditional mathematical model. In the next step, based on other neural networks, we will build a multi-dimensional spatial orbit model, and replace time series data with time series 3D pictures for research and analysis.

\section{Acknowledgements}

This work is supported by the SpectroLive Project form CNRS foundation.

\section{References}

[1] Cang ZY. Accuracy improvement and application of orbit prediction for low orbit space targets [D]. Nanjing: Nanjing University of Information Technology, 2016.

[2] Ma X, Tao Z, Wang Y, et al. Long Short-term Memory Neural Network for Traffic Speed Prediction using Remote Microwave Sensor Data[J]. Transportation Research Part C Emerging Technologies, 2015, 54:187-197.

[3] Hochreiter S, Schmidhuber J. Long short-term memory[J]. Neural Computation, 1997, 9(8):17351780 .

[4] Ma X, Tao Z, Wang Y, et al. Long short-term memory neural network for traffic speed prediction using remote microwave sensor data[J]. Transportation Research Part C: Emerging Technologies, 2015, 54: 187-197.

[5] Fusco G, Colombaroni C, Isaenko N. Short-term speed predictions exploiting big data on large urban road networks[J]. Transportation Research Part C: Emerging Technologies, 2016, 73: 183-201.

[6] Cong Y, Wang J, Li X. Traffic flow forecasting by a least squares support vector machine with a fruit 
fly optimization algorithm[J]. Procedia Engineering, 2016, 137(1): 59-68.

[7] Tang J, Liu F, Zou Y, et al. An improved fuzzy neural network for traffic speed prediction considering periodic characteristic[J]. IEEE Transactions on Intelligent Transportation Systems, 2017, 18(9): 2340-2350. 